\title{
A novel putative interactor for the low density lipoprotein receptor cytoplasmic domain
}

\author{
SALVATORE COSTA ${ }^{1}$, ALDO NICOSIA ${ }^{1}$, MARIA ANTONIETTA RAGUSA ${ }^{1}$, ANGELO BALDASSARE CEFALÙ ${ }^{2}$, \\ DANIELA POLLACCIA ${ }^{2}$, DAVIDE NOTO ${ }^{2}$, MAURIZIO AVERNA $^{2}$ and FABRIZIO GIANGUZZA ${ }^{1}$
}

\author{
Departments of ${ }^{1}$ Cellular and Developmental Biology 'A. Monroy', and ${ }^{2}$ Clinical Medicine of Emerging Diseases, \\ University of Palermo, I-90128 Palermo, Italy
}

Received December 14, 2009; Accepted January 18, 2010

DOI: $10.3892 / \mathrm{mmr} 00000263$

\begin{abstract}
Familial hypercholesterolemia (FH) is a genetic disease mainly caused by mutations in the low density lipoprotein receptor (LDL-R) gene. However, FH-like phenotypes may also arise from mutations occurring in other genes, the products of which normally interact with the LDL receptor. Although several FH-associated proteins have been discovered, many FH-like phenotypes cannot be linked to mutations in already characterized genes, suggesting the existence of other genes still to be identified, the mutations of which may be directly linked to the FH disorder. In order to identify new putative LDLr interactors possibly involved in its internalization and/or sorting, the cytoplasmic tail of the receptor was used as 'bait' in a two-hybrid assay. We identified an 85-amino acid protein able to bind the LDLr intracellular domain through the last $14 \mathrm{C}$-terminal amino acids. The novel protein is probably derived from the translation of an alternative open reading frame of the human MT2A gene.
\end{abstract}

\section{Introduction}

The LDL receptor (LDL-R) is the founding member of a larger class of related transmembrane receptors. These proteins combine three types of extracellular modules in different arrangements to carry out a wide range of physiologic functions, both as transport proteins and receptors/co-receptors in signal transduction cascades.

LDLr is the protein primarily responsible for the cell uptake of cholesterol-carrying particles from the blood. Human mutations that prevent LDL clearance lead to familial

Correspondence to: Professor Fabrizio Gianguzza, Department of Cellular and Developmental Biology 'A. Monroy', University of Palermo, Viale delle Scienze, Parco d'Orleans Ed. 16, I-90128 Palermo, Italy

E-mail: gianfab@unipa.it

Key words: lipoprotein receptors, low density lipoprotein receptor interactors, two-hybrid assay hypercholesterolemia (FH), an autosomal dominant disorder characterized by elevated plasma LDL-cholesterol levels and an increased risk of atherosclerosis $(1,2)$.

Three additional genes involved in LDL metabolism encode interactors of LDL-R and give rise to $\mathrm{FH}$ phenotypes. Familial defective apolipoprotein B-100 (FDB) is a syndrome clinically similar to FH caused by a few mutations in the gene encoding apolipoprotein B-100 (Apo-B100), which compromises the ability of the corresponding protein to interact with LDL-R.

A co-dominant form of hypercholesterolemia, autosomal dominant hypercholesterolemia type 3 (HCHOLA3), was recently described and was found to result from mutations in the gene encoding pro-protein convertase subtilisin kexin 9 (PCSK9), a protease of the subtilisin-like convertase family, which destroys low density lipoprotein receptors in the liver and thereby controls the level of LDL in plasma $(3,4)$. Finally, increasing evidence indicates the existence of a recessive form of severe hypercholesterolemia, phenotypically similar to homozygous FH but not linked to the LDL-R gene. This disorder, autosomal recessive hypercholesterolemia (ARH), is caused by mutations in the ARH gene, which encodes an adaptor protein required for normal LDL receptor-mediated endocytosis in hepatocytes (5-7).

Notably, PCSK9-mediated degradation of the LDL-R does not take place on the cell surface. Rather, the PCSK9-mediated degradation of the LDL-R appears to occur intracellularly, even when endocytosis through clathrin-coated pits is blocked by hypertonic medium (4). This finding suggests the existence of a novel ARH-independent pathway for LDLr internalization, which may involve unknown proteins.

The LDL-R gene family has traditionally been regarded as a class of constitutively recycling cell surface receptors mediating the endocytosis and lysosomal delivery of different ligands. In addition, lipoprotein receptors play important roles as activators of intracellular signaling cascades. Recently, the cytoplasmic adaptor or scaffold protein disabled-1 (DAB1) and FE65 were reported to interact with the cytoplasmic tails of certain LDL receptor family members $(8,9)$, thus connecting transmembrane receptors with endocytosis machinery and supporting the assembly of multi-protein complexes involved in signaling pathways. All these functions are likely mediated by protein-protein interactions, most of which are not yet 
known. In order to gain new insights regarding LDL-R interactors, we investigated the existence of unknown proteins able to bind the LDL-R cytoplasmic domain using both genetic (two-hybrid assay) and biochemical (co-immunoprecipitation assay) approaches $(10,11)$.

\section{Materials and methods}

Bait plasmid construct. The cDNA portion which encodes the LDL receptor whole cytoplasmic domain (LDL-R/CD) was amplified using the following primers: 5'-GAATTC CTATGGAAGAACTGGCGGC-3' and 5'-GTCGACGCAGAT GTTCACGCCACGTC-3'.

The 159-bp product was cloned as an EcoRI-SalI fragment into the EcoRI-SalI sites of the pGBKT7 2- $\mu$ TRP marked vector (Clontech, UK), downstream of the Gal4 DNA binding domain to produce a Gal4BD-LDL-R/CD fusion protein. The expression used by the vector contains the $A D H 1$ promoter, an SV40 T-antigen nuclear localization sequence, the c-myc epitope tag and the Gal4BD (residues 1-147). The final construct was verified by nucleotide sequencing.

Prey plasmid constructs. MT2AaORF cDNA was subcloned into the NdeI/XhoI sites of the pGADT7 2- $\mu$ LEU2 marked vector (Clontech, UK) downstream of the Gal4 DNA activation domain to produce a Gal4AD-MT2AaORF fusion protein. The pGADT7 vector contains the $A D H 1$ promoter, an SV40 T-antigen nuclear localization sequence, the hemagglutinin (HA) epitope and the Gal4AD (residues 768-881). MT2AaORF C-terminal deletion constructs were generated by PCR amplification using the following primers: 5'-CTATT CGATGATGAAGATACCCCA-3' (forward primer); 5'-ATA CTCGAGTGCAGGAGTCACGGCGG-3' (reverse, for the Gal4AD-MT $_{12}$ construct); 5'-ATACTCGAGCCTGGGCACT TGGCACAG-3' (reverse, for the Gal4AD-MT ${ }_{46}$ construct); 5'-ATACTCGAGTACATCTGGGAGCGGGG-3' (reverse, for the Gal4AD-MT ${ }_{71}$ construct).

The three products were independently cloned into the NdeI/XhoI site of the pGADT7AD vector, previously linearized with the same enzymes. All of the constructs were verified by nucleotide sequencing.

Description of the yeast strains. Two different Saccharomyces cerevisiae yeast strains were used in this study. AH109 strain: MATa trp1-901, leu2-3, 112, ura3-52, his3-200, gal4A, gal804, LYS2::GAL1 $1_{\mathrm{UAS}^{-}}-G A L 1_{\mathrm{TATA}^{-}}-H I S 3, \quad G A L 2_{\mathrm{UAS}^{-}}-G A L 2_{\mathrm{TATA}^{-}}-A D E 2$ URA3::MEL1 $1_{\mathrm{UAS}}-M E L 1_{\mathrm{TATA}}-$ lacZ MEL1. Y187 strain: MATa, ura3-52, his3-200, ade2-101, trp1-90, leu2-3, 112, gal4A, gal804, met, URA3::MEL1 $1_{\mathrm{UAS}}-M E L 1_{\mathrm{TATA}}$-lacZ MEL1.

Yeasttransformation and the two-hybridassay. Saccharomyces cerevisiae host strains AH109 MATa and Y187 MATa were grown at $30^{\circ} \mathrm{C}$ in YPD medium for $18 \mathrm{~h}$ and utilized for BD and $\mathrm{AD}$ fusion construct transformations, respectively, using the LiAc procedure as suggested by the supplier (Clontech Yeast Protocols Handbook, UK). Appropriate SD medium plates, SD/-Trp or SD/-Leu, were used to select AH109 MATa Gal4BD-LDL-R/CD-expressing trasformants and Y187 MATa Gal4AD-MT2AaORF-, Gal4AD-MT ${ }_{12}{ }^{-}, G_{\text {Gal4AD-MT }}$ - $^{-}$and Gal4AD-MT ${ }_{71}$-expressing transformants, respectively.
The GAL4BD-LDL-R/CD fusion protein was used as bait for screening a human liver Matchmaker Gal4 yeast twohybrid library (Clontech). Cells were allowed to mate at $30^{\circ} \mathrm{C}$ for $24 \mathrm{~h}$ in $2 \mathrm{X}$ YPDA/Kan $50 \mu \mathrm{g} / \mathrm{ml}$ as described in the manufacturer's instructions, and the mating mixture was plated on the appropriate SD medium (SD/-Ade/-His/-Leu/-Trp). Colonies grown on selective media and scored as positive for an interaction with the LDL-R/CD were tested for MEL1 reporter gene expression using $\mathrm{SD} /$-Ade/-His/-Leu/-Trp plates containing X- $\alpha$-Gal $40 \mu \mathrm{g} / \mathrm{ml}$. Moreover, the $\alpha$-galactosidase units were determined in a spectrophotometer assay by recording the $\mathrm{OD}_{410}$ value in the presence of PNP- $\alpha-$ Gal solution, as suggested by the manufacturer. Positive clones were analyzed by PCR using AD vector-specific primers, and were directly sequenced.

Production of tagged proteins. ${ }^{35} \mathrm{~S}$-methionine-labelled MT2AaORF HA-tagged and LDL-R/CD c-myc-tagged proteins were produced by in vitro translation from pGADT7-MT2AaORF and pGBKT7-LDL-R/CD constructs, respectively, using the TnT coupled Reticulocyte Lysate Systems (Promega). Transcription/translation reactions were performed in $50 \mu \mathrm{l}$ using $1 \mu \mathrm{g}$ DNA template in the presence of 1 unit of T7 RNA polymerase (Promega) and $2 \mu 1\left({ }^{35} \mathrm{~S}\right)$ methionine $(>1,000 \mathrm{Ci} / \mathrm{mmol}$ at $10 \mathrm{mCi} / \mathrm{ml})$ (GE HealthcareAmersham) for $1.5 \mathrm{~h}$ at $30^{\circ} \mathrm{C}$.

Physical interaction of MT2AaORF with the cytoplasmic domain of the LDL receptor. Labelled MT2AaORF HA-tagged and LDL-R/CD c-myc-tagged proteins (10 $\mu 1$ each) were mixed together and incubated at room temperature for $1 \mathrm{~h}$. One microgram of either the anti-c-Myc monoclonal antibody or anti-HA-Tag polyclonal antibody (Clontech) were added into two separate reactions. In parallel, MT2AaORF HA-tagged $(10 \mu \mathrm{l})$ and LDL-R/CD c-myc-tagged $(10 \mu \mathrm{l})$ proteins were incubated with $1 \mu \mathrm{g}$ of anti-c-Myc monoclonal antibody and anti-HA-Tag polyclonal antibody (Clontech), respectively, as negative controls, to evaluate antibody specificity. After a 1-h incubation, $3 \mu \mathrm{l}$ of PBS-washed Protein A beads were added, and adequate mixing was ensured. Protein A beads were washed 5 times with wash buffer as recommended by the supplier, and were finally resuspended in 20 $\mu \mathrm{l}$ SDS-PAGE loading buffer. Bound proteins were eluted and denatured under reducing conditions by heating the beads at $80^{\circ} \mathrm{C}$ for $5 \mathrm{~min}$, and were then analyzed by SDS-PAGE. The dried gel was finally exposed to X-ray film (Amersham Hyperfilm MP) overnight at room temperature.

\section{Results}

Two-hybrid screening assay. In order to identify novel interactors of the LDL receptor cytosolic domain (LDL-R/CD), we used the Gal4 system-based yeast two-hybrid assay method (12). A Matchmaker Gal4 human liver cDNA library, made in the pACT2 AD vector, was screened with the C-terminal intracellular domain (from amino acid 788 to 839) of the human LDL receptor (LDL-R/CD). To this end, a recombinant construct was prepared as described in the Materials and methods and introduced into the Saccharomyces cerevisiae host strain AH109 in order to stably express LDL-R/CD in 


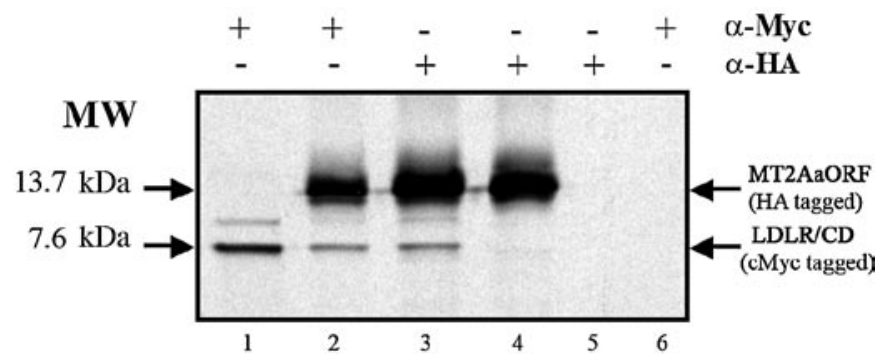

Figure 1. Co-immunoprecipitation of MT2AaORF and LDL-R/CD by either anti-c-Myc $(\alpha-M y c)$ monoclonal antibody or anti-HA tag polyclonal antibody $(\alpha-\mathrm{HA})$. The immunoprecipitates were analyzed by SDS-PAGE. Lane 1 : LDL-R/CD immunoprecipitated with $\alpha$-Myc; Lane 2: mix of LDL-R/CD and MT2AaORF, immunoprecipitated with $\alpha$-Myc; Lane 3: mix of LDL-R/ $\mathrm{CD}$ and MT2AaORF, immunoprecipitated with $\alpha-\mathrm{HA}$; Lane 4: MT2aORF immunoprecipitated with the $\alpha-\mathrm{HA}$; Lane 5: LDL-R/CD immunoprecipitated with $\alpha$-HA; Lane 6: MT2AaORF immunoprecipitated with $\alpha$-Myc.

yeast, as a fusion protein with the GAL4 DNA-binding domain (from amino acid 1 to 147). We verified that the production of the fusion product did not affect mating efficiency, and evaluated the intrinsic transcription activation of the reporter genes (data not shown).

The two-hybrid assay was performed by the yeast 'mating protocol' using the AH109-MATa GAL4BD-LDL-R/CD fusion protein-expressing cells and the Y187-MAT $\alpha$ cells, pre-transformed with the human cDNA liver library. The mating mixture was seeded on plates containing either leucine and tryptophan-lacking (non-selective) medium or adenosine, histidine, tryptophan and leucine-lacking (selective) medium. Screening of $1 \times 10^{6}$ independent library clones allowed identification, on selective media, of 350 diploid clones, which scored positive for an interaction with LDL-R/CD and were characterized by different degrees of mell reporter gene activation. The AD plasmids were recovered from diploid cells, and the inserts were sequenced: 108 independent insert sequences ( $31 \%$ of the total colonies) showed full homology with the human metallothionein 2A cDNA (NM_005953) in a BLAST search at the National Center for Biotechnology Information, whereas the remaining low frequency clones were classified as probable false positives. However, upon analyzing the amino acid sequence of the isolated fusion proteins, we realized that none of the clones encoded the expected MT2A protein. Since yeast can tolerate translational frameshifts, an ORF in the wrong reading frame may correspond to the protein responsible for the interaction. To verify this, we cloned the cDNA insert in the right frame for MT2A and determined whether the ADE2, HIS3 and MEL1 reporters remained active in the presence of the GAL4BDLDL-R/CD fusion bait protein. Diploid cells were seeded both onto $\mathrm{SD} /$-Leu/-Trp plates to gain positive controls, and onto SD/-Ade/-His/-Leu/-Trp plates to investigate reporter gene activation. No viable diploid cells were observed on the selective media, indicating that the protein responsible for the interaction with LDL-R/CD is encoded by an alternative reading frame of the MT2A gene, which we named MT2A alternative ORF (MT2AaORF). The specificity of the LDL-R/CD-MT2AaORF interaction in the yeast two-hybrid assay was confirmed by transformation of the positive prey cDNA clone into yeast AH 109 cells, together with vectors expressing the GAL4 DNA-binding domain (GAL4BD) alone or the GAL4BD-LDL-R/CD. Only yeast cells containing the Gal4AD-MT2AaORF and the GAL4BD-LDL-R/CD plasmids displayed an interaction phenotype, giving rise to the reporter gene activation, whereas the empty GAL4BD was negative in these control transformation assays (data not shown).

$M T 2 A a O R F$ interacts with $L D L-R / C D$ in vitro. To verify the interaction between LDL-R/CD and MT2AaORF at the biochemical level, co-immunoprecipitation experiments were performed. Full-length MT2AaORF was subcloned into a vector allowing the $\mathrm{T} 7$ promoter-driven expression of MT2AaORF as a radiolabelled HA-tagged fusion protein by an in vitro coupled transcription-translation reaction. The HA-MT2AaORF (13.7 kDa) was then mixed with a $7.6-\mathrm{kDa}$ c-Myc epitope-tagged LDL-R/CD (c-Myc-LDL-R/CD), obtained by means of the in vitro transcription-translation reaction. The protein mixture was incubated with either an anti-c-Myc polyclonal or an anti-HA monoclonal antibody in two separate reactions, and the immune complexes were analyzed by autoradiography after SDS-PAGE (Fig. 1). The anti-c-Myc monoclonal antibody was found to immunoprecipitate not only c-Myc-LDL-R/CD but also the radiolabelled HA-MT2AaORF (Fig. 1, lanes 1 and 2); on the other hand, the anti-HA polyclonal antibody was able to immunoprecipitate not only HA-MT2AaORF but also the radiolabelled c-Myc-LDL-R/CD (Fig. 1, lanes 3 and 4), while no cross immunoreactions were observed (Fig. 1, lanes 5 and 6). Since both antibodies were able to immunoprecipitate both proteins, we deduced that MT2AaORF is indeed capable of interacting with LDL-R/CD, in accordance with the data obtained by the yeast two-hybrid assay.

In silico MT2AaORF characterization. Proteomics computational tools were applied to find out and characterize potential structures or domains of the MT2AaORF. Formal queries regarding the protein sequence with popular domain detection algorithms (Pfam, SMART, ELM and Interproscan, ExPASy proteomics server of the Swiss Institute of Bioinformatics) (13-16) predicted that the 85 -aa MT2AaORF protein has a molecular mass of $8.46 \mathrm{kDa}$ and contains at least three putative functional domains: i) an alanine-rich region profile (aa 5-66), ii) a proline-rich region (aa 3-45) organized in three SH3 docking sites (aa 3-9, aa 6-12, aa 39-45), and iii) a $\beta$ and $\gamma$-crystallin domain (aa 77-85) folded into a Greek key motif which includes an SH2-PTB docking site (aa 82-85) (Fig. 2A).

$M T 2 A a O R F-L D L-R / C D$ interaction. In order to identify the protein region/domain responsible for the interaction with the LDL-R/CD, three deletion clones were constructed, each of which lacks different portions of the C-end. To accomplish this, suitable modified primer pairs were utilized to amplify three different regions of the MT2AaORF cDNA, which, after cloning and translation, gave rise to truncated peptides of 71, 46 and 12 aa, respectively (the amino acid sequences of these deleted proteins are provided in Fig. 2B). The three PCR products were then inserted into the vector pGADT7, in frame with the sequence which encodes the GAL4 activation domain. The constructs were respectively named: 
A

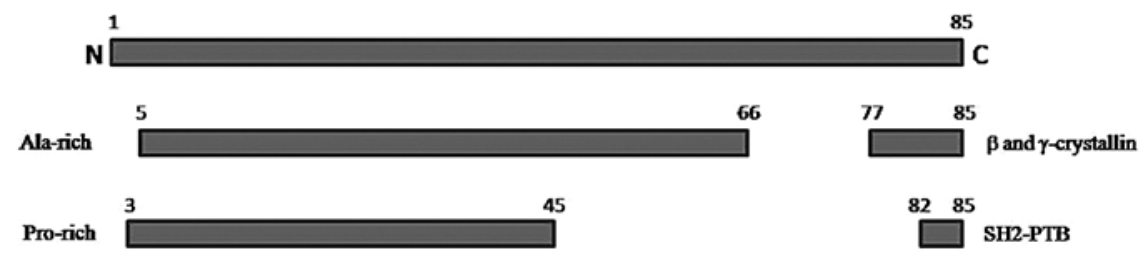

B

\begin{tabular}{|l|}
\hline HA+MT2AOORF \\
MEYPYDVPDYAMAMEAPGIRIRGRVDQVPANPRATCPDSSRLFSSPWIPTAPAPPVTPAPAPAPANAKSANAPP \\
ARKAAAPAALWVPSVPRAASAKGRRTSAAAAPDGTAPLPDVKNATSQTWIFYVQP \\
HA+MT12 \\
MEYPYDVPDYAHMAMEAPGIRIRGRVDQVPANPRATCPDSSRLFSSPWIPTAPAPPVTPA \\
HA+MT46 \\
MEYPYDVPDYAHMAMEAPGIRIRGRVDQVPANPRATCPDSSRLFSSPIIPTAPAPPVTPAPAPAPANAKSANAPP \\
ARKAAAPAALWAVPSVR \\
HA+MT71 \\
MEYPYDVPDYAHMAMEAPGIRIRGRVDVVANPRATCPDSSRLFSSPIPTAPAPPVTPAPAPAPANAKSANAPP \\
ARKAAAPAALWVPSVPRAASAKGRRTSAAAAPDGTAPLPDV
\end{tabular}

Figure 2. Summary of the putative features of the MT2AaORF ( 85 aa) as indicated by Expasy proteomic tools (A), and amino acid sequence of the truncated fusion proteins encoded by the subclones obtained by amplification of three different regions of the cDNA (B). Numbers indicate amino acid positions with respect to the full-length protein ( $85 \mathrm{aa})$.

A

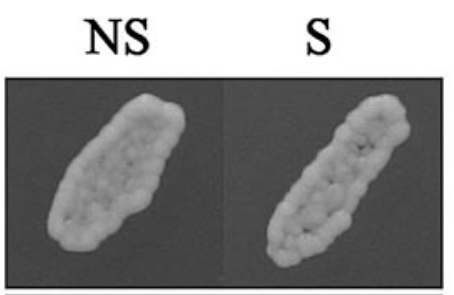

B

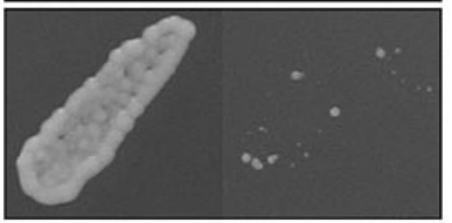

C

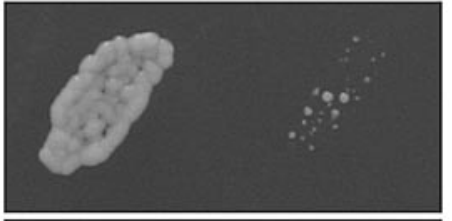

D

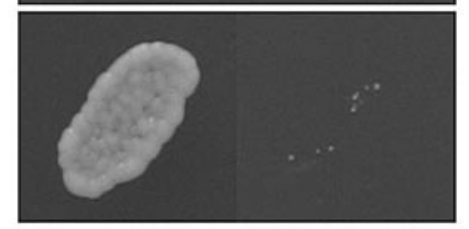

Figure 3. Growth of yeast diploids on SD selective (-hys, -leu, -trp) (S) and non-selective media (-leu, -trp) (NS) after mating. (A) pGBKT7-LDL-R/CD

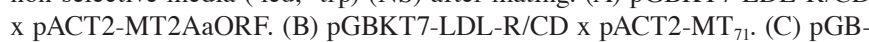
KT7-LDL-R/CD x pACT2-MT 46 . (D) pGBKT7-LDL-R/CD x pACT2-MT ${ }_{12}$.

i) Gal4AD-MT ${ }_{12}$, which includes only two SH3 docking sites at the N-terminus; ii) Gal4AD-MT $\mathrm{M}_{46}$, which contains the entire proline-rich region, organized into three $\mathrm{SH} 3$ docking sites, and iii) Gal4AD-MT $\mathrm{MT}_{71}$, which lacks the $\mathrm{SH} 2$ docking site contained in the Greek Key motif. The constructs and the original pACT2AD-MT2AaORF were transformed into the Y187 MAT $\alpha$ cells and tested in a direct two-hybrid assay with the GAL4BD-LDL-R/CD fusion protein-expressing AH109MATa cells. After mating and plating the diploid cells onto selective media, we observed that none of the deleted clones produced colonies on selective media, while the full length MT2AaORF clone showed an interaction phenotype able to activate the reporter genes (Fig. 3). These results strongly suggested that the MT2AaORF binding site is located in the $14 \mathrm{C}$-terminal residues of the protein and that the MT2AaORF-LDL-R/CD interaction is supported by the SH2 ligand functional site class.

\section{Discussion}

In the present study, the yeast two-hybrid assay, based on the mating approach, was used to identify putative proteins capable of interacting with the cytoplasmic domain of the LDL receptor (LDL-R/CD). We identified 108 recombinant clones, all of which contain the same MT2A gene alternative reading frame previously reported in the literature as CES1/MT2A (GenBank M26637.1) (17). The encoded protein is able to interact with the LDL-R/CD. The MT2AaORF-LDL-R/CD interaction, identified by a genetic approach, was also confirmed at the biochemical level by co-immunoprecipitation assays.

The MT2AaORF encodes an 85-amino acid-long protein, the sequence of which is shown in Fig. 2B (upper row). When used as a query in diverse domain detection algorithms for a computational analysis, the novel protein showed different putative functional sites. At least four putative protein-protein interaction sites spanning the whole amino acid sequence were recognized. In particular, in silico analyses, performed at SIB, revealed in the $\mathrm{C}$-end of the protein some motifs distantly related to protein/protein interaction motifs, such as a generic motif for glycosylation (aa 72-77) and a target site for phosphorylation (aa 73-79). A more interesting motif (aa 82-85) belongs to the SH2 ligand-binding functional site class. SH2 and PTB domains are small protein modules, 
used as key building blocks in the pathways, which allow intracellular transduction of extracellular signals to different compartments of the living cells. Although both domains were initially identified as modules that recognize phosphorylated tyrosines, subsequent studies indicated that only $\mathrm{SH} 2$ binding to their target proteins is strictly regulated by tyrosine phosphorylation, whereas most PTB domains constitutively bind to their (non-phosphorylated) targets (18).

In the present study, the yeast two-hybrid system was also used to identify and map the MT2AaORF region, which directly interacts with the LDL-R/CD. The assays performed with C-terminal progressive deletion constructs of MT2AaORF as prey suggested that the last $14 \mathrm{C}$-terminal amino acid residues of the protein, which include an SH2-PTB docking site, are responsible for the interaction with LDL-R/CD.

Finally, although none of the already characterized motifs known to interact with the LDLr internalization signal (FDNPVY) were identified in the MT2AaORF, our results confirmed that the LDL-R uses different internalization pathways depending on the ligand (19-21).

Notably, a subtilisin/kexin cleavage site was present in the protein, together with the SH2-PTB functional site. This finding could provide new insights into LDL-R regulation. PCSK9, a subtilisin/kexin family member, is indeed known to bind LDL-R, thus promoting its degradation via the ARH adaptor function, a protein that contains a PTB motif (22).

Evidence was reported by other authors that PCSK9mediated LDL-R degradation may occur without ARH involvement, thus suggesting the existence of a novel ARH-independent pathway for PCSK9 activity on LDL-R (4).

In light of the present results derived from genetic, biochemical and bioinformatic studies, a functional and physiological role of MT2AaORF can be suggested, possibly related to its putative involvement in LDL-R internalization and degradation, as suggested by its ability to interact with the cytoplasmic domain and, in particular, with the SH2, PTB motif.

The possibility of an in vivo MT2AaORF involvement in the LDL-R pathway, if further confirmed, also allows consideration of MT2AaORF as a novel pharmacological target in FH therapy.

\section{Acknowledgements}

We wish to thank Professor I. Di Liegro for suggestions and the critical reading of the manuscript. This study was supported by the University of Palermo ('ex 60\%' grants from Università degli Studi di Palermo to F.G. and M.A.), Italy.

\section{References}

1. Soutar AK and Rossi Naoumova P: Mechanisms of disease: genetic causes of familial hypercholesterolemia. Nat Clin Pract Cardiovasc Med 4: 214-225, 2007.

2. Bengoechea-Alonso M and Ericsson J: SREBP in signal transduction: cholesterol metabolism and beyond. Curr Opin Cell Biol 19: 215-222, 2007.

3. Horton JD, Cohen JC and Hobbs HH: Molecular biology of PCSK9: its role in LDL metabolism. Trends Biochem Sci 32: 71-77, 2006.
4. Holla $\varnothing \mathrm{L}$, Cameron J, Berge KE, Ranheim T and Leren TP: Degradation of the LDL receptors by PCSK 9 is not mediated by a secreted protein acted upon by PCSK9 extracellularly. BMC Cell Biol 8: 9, 2007.

5. Pisciotta L, Priore Oliva C, Mario Pes G, Di Scala L, Bellocchio A, Fresa R, Cantafora A, Arca M, Calandra S and Bertolini S: Autosomal recessive hypercholesterolemia (ARH) and homozygous familial hypercholesterolemia $(\mathrm{FH})$ : a phenotypic comparison. Atherosclerosis 188: 398-405, 2006.

6. Abera B, Marais AD, Raal FJ, Leisegang F, Jones S, George P and Henderson HE: Autosomal recessive hypercholesterolaemia: discrimination of $\mathrm{ARH}$ protein and LDLR function in the homozygous FH phenotype. Clin Chim Acta 378: 33-37, 2007.

7. Van Hoof D, Rodenburg KW and van der Horst DJ: Intracellular fate of LDL receptor family members depends on the cooperation between their ligand binding and EGF domains. J Cell Sci 118: 1309-1320, 2005.

8. Stolt PC and Bock HH: Modulation of lipoprotein receptor functions by intracellular adaptor proteins. Cell Signal 18: 1560-1571, 2006.

9. Pietrzik CU, Yoon IS, Jaeger S, Busse T, Weggen S and Koo K: FE65 constitutes the functional link between the low-density lipoprotein receptor-related protein and the amyloid precursor protein. J Neurosci 24: 4259-4265, 2004.

10. Aloy P and Russell RB: The third dimension for protein interactions and complexes. Trends Biochem Sci 27: 633-638, 2002.

11. Stephens DJ and Banting G: The use of yeast two-hybrid screens in studies of protein: protein interactions involved in trafficking. Traffic 1: 763-768, 2000.

12. Fields S and Song O: A novel genetic system to detect proteinprotein interactions. Nature 340: 245-247, 1989.

13. Finn RD, Tate J, Mistry J, Coggill PC, Sammut JS, Hotz HR, Ceric G, Forslund K, Eddy SR, Sonnhammer EL and Bateman A: The Pfam protein families database. Nucleic Acids Res 36: D281-D288, 2008.

14. Schultz J, Milpetz F, Bork P and Ponting CP: SMART, a simple modular architecture research tool: identification of signaling domains. Proc Natl Acad Sci USA 95: 5857-5864, 1998.

15. Puntervoll P, Linding R, Gemünd C, et al: ELM server: a new resource for investigating short functional sites in modular eukaryotic proteins. Nucleic Acids Res 31: 3625-3630, 2003.

16. Quevillon E, Silventoinen V, Pillai S, Harte N, Mulder N, Apweiler R and Lopez R: InterProScan: protein domains identifier. Nucleic Acids Res 33: W116-W120, 2005.

17. Duguid JR, Bohmont CW, Liu NG and Tourtellotte WW: Changes in brain gene expression shared by scrapie and Alzheimer disease. Proc Natl Acad Sci USA 86: 7260-7264, 1989.

18. Schlessinger $\mathrm{J}$ and Lemmon MA: $\mathrm{SH} 2$ and PTB domains in tyrosine kinase signaling. Sci STKE 191: 12, 2003.

19. Gotthardt M, Trommsdorf M, Nevitt MF, Sheltoni J, Richardsoni JA, Stockinge W, Nimpf J and Herz J: Interactions of the low density lipoprotein receptor gene family with cytosolic adaptor and scaffold proteins suggest diverse biological functions in cellular communication and signal transduction. J Biol Chem 275: 25616-25624, 2000.

20. Stockinger W, Sailler B, Strasser V, Recheis B, Fasching D, Kahr L, Schneider WJ and Nimpf J: The PX-domain protein SNX17 interacts with members of the LDL receptor family and modulates endocytosis of the LDL receptor. EMBO J 21: 4259-4267, 2002.

21. Michaely P, Zhao Z, Li WP, Garuti R, Huang LJ, Hobbs HH and Cohen JC: Identification of a VLDL-induced, FDNPVYindependent internalization mechanism for the LDLR. EMBO J 26: 3273-3282, 2007.

22. Zhang DW, Lagace TA, Garuti R, Zhao Z, McDonald M, Horton JD, Cohen JC and Hobbs HH: Binding of proprotein convertase subtilisin/kexin type 9 to epidermal growth factorlike repeat A of low density lipoprotein receptor decreases receptor recycling and increases degradation. J Biol Chem 282: 18602-18612, 2007. 